Int. J. Odontostomat., 8(1):99-105, 2014.

\title{
Contaminación Bacteriana Generada por Aerosoles en Ambiente Odontológico
}

\author{
Analysis of Bacterial Contamination Produced by \\ Aerosols in Dental Clinic Environments
}

\author{
María Fabiola Bustamante Andrade*; Jessica Herrera Machuca*; \\ ${\text { Roxana Ferreira } \text { Adam }^{* * *} \text { \& Denisse Riquelme Sanchez }}^{* * *}$
}

BUSTAMANTE, A. M. F.; HERRERA, M. J.; FERREIRA, A. R. \& RIQUELME, S. D. Contaminación bacteriana generada por aerosoles en ambiente odontológico. Int. J. Odontostomat., 8(1):99-105, 2014.

RESUMEN: En odontología estamos expuestos a muchos microorganismos por la producción de aerosoles. Por ello es esencial conocer la naturaleza de éstos y su potencial patogénico. El objetivo de esta investigación es determinar contaminación bacteriana, generada por aerosoles durante procedimientos odontológicos, con uso de pieza de mano de alta velocidad, realizados por alumnos de la carrera, en Clínica Odontológica Docente Asistencial (CODA), Universidad de La Frontera, Temuco, Chile. Estudio con muestra aleatoria, de 16 de un total de 32 unidades dentales, estratificado por box, con 40 muestras, ocho placas control y 32 placas prueba. El medio de cultivo, se mantuvo por diez minutos, donde se realizaron acciones de operatoria con turbina, bajo aislamiento absoluto, ubicadas en frente del operador y pechera del paciente. Las muestras fueron analizadas microscópicamente, incubadas a $37^{\circ} \mathrm{C}$ en atmósfera de oxígeno por 24 horas y dióxido de carbono a las 48 horas. Treinta y dos placas prueba fueron positivas, registrándose diversidad de crecimiento bacteriano, promedio 58,874 Unidad Formadora de Colonias (UFC) por unidad dental. El mayor porcentaje de microorganismos fueron: Bacillus spp. $(28,56 \%)$ y Bacilos Gram positivos $(24,31 \%)$. Siete placas control resultaron negativas y una con 3 UFC de Micrococcus spp. La mayoría de los microorganismos encontrados son comensales potencialmente patógenos. Al comprobar que los aerosoles constituyen una fuente importante de emisión de microorganismos, se hace imprescindible cumplir con todas las normas de bioseguridad que protegen tanto al operador como al paciente.

PALABRAS CLAVE: ambiente odontológico, contaminación bacteriana, aerosoles, turbina.

\section{INTRODUCCIÓN}

El odontólogo como profesional de la salud, está expuesto a una gran cantidad de microorganismos, provenientes de la sangre, secreciones orales y respiratorias del paciente, pudiendo ser agentes de enfermedades infecciosas. El hombre vive en simbiosis y equilibrio con un gran número de microorganismos, pero cuando éste se altera, desafían los mecanismos de defensa de sus huéspedes y causan daño (Cascone et al., 2002). La posibilidad de infectarse y enfermar, denominada riesgo, es directamente proporcional a la frecuencia de exposiciones a los agentes infecciosos, pueden ser vehiculizados por instrumental, aire, agua, saliva y/o sangre (Molinares, 1993). Este riesgo es variable y se relaciona al grado de formación de aerosoles; la generación de campos sangrantes y salpicaduras que puedan tener contacto directo o indirecto con mucosa nasal, oral, conjuntival, y/o lesiones cutáneas; como también, el riesgo de cortes y punciones (de Lucas Tomas et al., 1982). La humedad y temperatura de la cavidad oral crean un amplio rango de habitats, con diferentes condiciones ambientales que provee un medio ideal para el crecimiento y colonización de microorganismos (Loesche, 1976). La microbiota oral es extremadamente compleja, se han llegado a aislar unas 200 especies distintas de microorganismos de una misma cavidad oral; la mayor parte de característica transitoria, quedando como residentes aproximadamente 20 especies (Checchi et al., 1991).

\footnotetext{
Enfermera Universitaria, Mg. en Ciencias Mención Morfología, Profesor Asistente, Departamento de Odontología Integral Adulto, Facultad de Odontología, Universidad de La Frontera, Temuco, Chile.

* Cirujano Dentista, Mg. en Educación Mención en Evaluación Educacional, Universidad Autónoma de Chile, Temuco, Chile.

* Cirujanos Dentistas, Temuco, Chile.
} 
La cavidad oral es un ecosistema abierto y dinámico, esta comunidad ecológica ha sido denominada Biofilm (Donlan, 2001), que corresponde a un conjunto de biomasa microbiana siendo una unidad sellada, englobada en polisacáridos extracelulares lo que los hace resistentes a las defensas del huésped y a los antibióticos tanto locales como sistémicos (de Beer, 1994). Los biofilms también se forman rápidamente en las líneas del agua de la unidad dental (Williams et al., 1993). Para cumplir con la legislación de salud y seguridad, es necesario instituir medidas de control de infección bajo el estándar de la American Dental Association. (ADA) estableciendo como aceptado la formación de menos de $200 \mathrm{UFC} / \mathrm{ml}$ de bacterias aeróbicas en el agua (Dreyer \& Hauman, 2001).

En los procedimientos odontológicos el uso de instrumental rotatorio y jeringa triple, crea un spray visible o aerosol que contiene principalmente gotas de agua, saliva, sangre, microorganismos, y otros desechos (Harrel \& Molinari, 2004; Hauman, 1994). La producción de aerosoles por el uso de piezas de mano de alta velocidad, scalers sónicos, ultrasónicos y jeringa triple está bien documentada en la literatura odontológica (Bentley et al., 1994; Sacchetti et al., 2006; Wood, 1992). Estos aerosoles precipitan por la gravedad quedando en las superficies, y las partículas pequen'as o microgotas quedan suspendidas en el aire por varias horas, constituyendo un riesgo, ya que pueden ser inhaladas (Shpuntoff \& Shpuntoff, 1993).

Algunos estudios han demostrado que el aerosol generado por el uso de la turbina dentro de la cavidad bucal, emite cerca de 1.000 unidades formadoras de colonias bacterianas, otros han reportado que los microorganismos se han encontrado a 1,80 mts. de la turbina en uso (Santiago et al., 1994). Las concentraciones más altas de microorganismos se encontraron a $60 \mathrm{~cm}$ enfrente al paciente. Se ha reportado que las bacterias generadas por el uso del limpiador ultrasónico (scaler) pueden permanecer en el aire por 24 horas (Pankhurst, 2003).

En un análisis clínico los microorganismos más comúnmente encontrados en el spray de aerosol fueron Streptococos, Diphteroides, Neisseria y Staphylococos. La Neisseria comensal es el más encontrado en los fluídos orales contaminados con saliva o mucosas, ellas son usualmente no patogénica (Shpuntoff \& Shpuntoff). Estos fluidos del paciente retenidos en las superficies internas de los componentes de las piezas de mano de alta y baja velocidad, puede ser expelido intraoralmente durante usos subsecuentes, lo que demuestra, una forma por la cual las bacterias pudieran ser incorporadas en la nube de aerosol que se forma cuando se usa la turbina (Prieto \& Maestre, 2003). Cualquier dispositivo dental conectado al sistema aire/agua que entra a la boca del paciente, incluyendo las piezas de mano de alta velocidad, debe ser accionados para descargar agua, aire o una combinación de ambos, por un mínimo de 20-30 segundos después del uso con cada paciente, con el agua se favorece la eliminación mecánica de residuos del paciente que pudieran entrar a la turbina y líneas de agua y aire (Winn et al., 1992; Grenier, 1995). Evidencia científica indica que los microorganismos están aún presentes en las superficies internas después de descargar agua en las piezas de mano por un período de cinco minutos (Santiago et al.; Sanclement et al., 2005).

El propósito de esta investigación es determinar la contaminación bacteriana de los aerosoles producidos durante los procedimientos odontológicos utilizando turbina de alta velocidad, realizados por alumnos en la CODA., de la Universidad de La Frontera y así demostrar el riesgo potencial que representan para ellos, para los pacientes, docentes, personal auxiliar y administrativo.

\section{MATERIAL Y MÉTODO}

Se realizó un estudio de corte transversal, cuasiexperimental durante los años 2003-2004, en las dependencias de la CODA-CDT del Hospital Hernán Henríquez Aravena, con alumnos de cuarto año de la Facultad de Odontología, Universidad de La Frontera, Temuco, Chile.

La población de estudio corresponde a 32 unidades dentales de CODA; la unidad de análisis el sillón dental y su entorno inmediato (radio de 1,10 metros). Fueron incluidos 32 sillones, distribuidos en cuatro box, con 8 sillones cada uno y los alumnos de cuarto año de la carrera que utilizaban cada unidad. Fueron excluidos los sillones sin uso por problemas técnicos. Para determinar el tamaño de la muestra se utilizó un nivel de confianza de 95\%, una desviación estándar (DE) de 56,56 (DE de UFC observada) y una precisión del $10 \%$, obteniendo una muestra representativa de 16 unidades ocupadas por 9 alumnos de sexo masculino y 7 de sexo femenino. La selección de la muestra se realizó mediante muestreo aleatorio simple, estratificado por box. 
Para determinar la presencia de bacterias en los aerosoles, se usaron placas de cultivo desechables con medio agar sangre, el cual fue elegido por sus características no selectivas y su habilidad de promover el crecimiento de muchos microorganismos. Se tomaron en 2 grupos de cultivo, uno control y otros prueba.

Los cultivos control se tomaron un día lunes (día 0), utilizando a 8 placas, dos por cada box. Para la toma de muestra, las placas se dejaron abiertas por $10 \mathrm{mi}-$ nutos, en sector central del box, desocupado, después de realizada la limpieza por personal auxiliar, entre las 13:00 y 13:30 h. Posteriormente, las placas se sellaron, rotularon y enviaron al laboratorio de microbiología, a temperatura ambiente, para ser incubadas por $48 \mathrm{~h}$ en total; $24 \mathrm{~h}$ en atmósfera normal y 24 horas en atmósfera $\mathrm{CO}_{2}$ y realizar recuento de UFC y la identificación de cada una.

Los cultivos prueba se tomaron 2 días después de la toma control. Se utilizaron 2 placas por cada unidad dental, ubicadas en dos sitios diferentes. Una placa, se ubicó en la frente del operador adosada por un cintillo, aproximadamente a $30 \mathrm{~cm}$ de fuente de emisión de aerosoles. La segunda placa se ubicó en la pechera del paciente, aproximadamente a $20 \mathrm{~cm}$ de la fuente de emisión de aerosoles (Fig. 1). En todos los casos, los operadores realizaron acciones de trabajo operatoria, bajo aislamiento absoluto, con uso de la turbina de alta velocidad. Las placas se encontraban cerradas al momento de ubicarlas en los sitios de análisis, y se abrie-

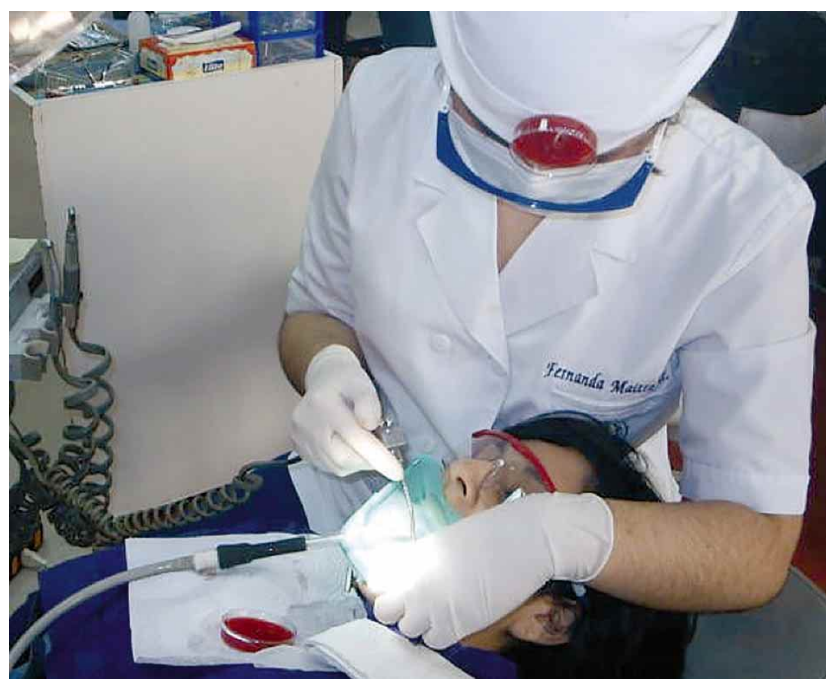

Fig. 1. Posicionamiento de las placas de cultivo prueba. Se utilizaron 2 placas por cada unidad dental, una ubicada en la frente del operador adosada por un cintillo, aproximadamente a $30 \mathrm{~cm}$ de fuente de emisión de aerosoles y otra en la pechera del paciente, aproximadamente a $20 \mathrm{~cm}$ de la fuente de emisión de aerosoles. ron al comenzar el uso de la turbina, permaneciendo expuestas por 10 minutos durante los cuales la turbina funcionaba a intervalos intermitentes. Luego las placas fueron selladas, rotuladas y enviadas al laboratorio para ser incubadas como se describió anteriormente.

En todos los casos, se realizó el recuento de UFC, y la identificación de cada colonia mediante sus características macroscópicas, microscópicas, tinción de Gram y pruebas de identificación. Se realizó un análisis exploratorio descriptivo de las variables bajo estudio, a través de tablas estadísticas.

\section{RESULTADOS}

Fueron adecuadamente procesadas 40 muestras, 8 cultivos de las placas control y 32 de las prueba. Entre las placas control, siete resultaron negativas y sólo una fué positiva con tres UFC de Micrococcus spp. Todas las placas prueba, resultaron positivas para crecimiento bacteriano, registrándose diversidad de crecimiento bacteriano, con una cantidad promedio de 58,874 UFC por sujeto.

Las especies de microorganismos encontradas se agruparon según su morfología y tinción de Gram en: Bacilos Gram (+) (Bacillus spp. y Corynebacterium spp.), Cocaceas Gram (+) (Micrococcus spp., Staphylococcus coagulasa negativa, Streptococcus spp. y Streptococcus viridans), Cocaceas Gram (-) (Neisseria spp.), y cocobacilos.

Del total de microorganismos encontrados el mayor porcentaje corresponde a Bacillus spp. $(28,56 \%)$ y Bacilos Gram (+) (24,31\%), representando más del $50 \%$ del total de microorganismos desarrollados. El microorganismo encontrado en menor porcentaje corresponde a Neisseria spp. $(0,42 \%)$ (Tabla I).

La mayor cantidad de microorganismos se registró en el Box 3, cercano al $36 \%$ del total de microorganismos. En orden decreciente el Box 1, registro un $31 \%$, el Box 4 , un $24 \%$ y el Box 2 un $9 \%$. De las nueve especies de microorganismos, cuatro alcanzaron sus mayores niveles en Box 1 (Corynebacterium spp., Micrococcus spp., Neisseria spp. y Streptococos viridans). En Box 3 se encontró la mayoría de los microorganismos Bacillus spp. (81\%) y Staphylococos coagulasa negativa. En Box 4 se registraron los más altos valores de Bacilos Gram (+), Cocobacilos Gram $(+)$ y Streptococcus spp. 
Tabla I. Distribución por especies de microorganismos en aerosoles, generados por uso de pieza de mano de alta velocidad, en la CODA, Universidad de La Frontera, Temuco, Chile.

\begin{tabular}{lcr}
\hline Especies & Frecuencia & $\%$ \\
\hline Bacillus spp. & 269 & 28,56 \\
Bacilos Gram (+) & 229 & 24,31 \\
Cocobacilos Gram (+) & 15 & 1,59 \\
Corynebacterium spp. & 19 & 2,02 \\
Micrococcus spp. & 141 & 14,97 \\
Neisseria spp. & 4 & 0,42 \\
Streptococos viridans & 202 & 21,44 \\
Stafilococos coagulasa (-) & 52 & 5,52 \\
Streptococcus spp. & 11 & 1,17 \\
Total & 942 & 100,00 \\
\hline
\end{tabular}

La cantidad de microorganismos encontrados en placas prueba de la Pechera, fue seis veces mayor que los encontrados en placas prueba de la Frente, excepto Cocobacilos Gram (+). Especies como Corynebacterium spp. y Neisseria spp. no presento desarrollo en placas de agar ubicadas en Frente (Tabla II).
Se encontró tres veces más microorganismos en mujeres que en hombres, excepto Cocobacilos Gram (+). Neisseria spp., se encontró sólo en individuos de sexo masculino (Tabla III).

La distribución de microorganismos según sexo y ubicación de las placas prueba, mantiene la tendencia descrita. Mayor cantidad de contaminación en mujeres y en pechera, excepto para Cocobacilos Gram positivo. Se observa que en mujeres la diferencia en cantidad de microorganismos encontrados en los lugares pechera y frente es marcadamente mayor que en hombres (relación 9/3 veces más en pechera).

\section{DISCUSIÓN}

De acuerdo a los resultados obtenidos en esta investigación podemos señalar que, existe una gran cantidad y diversidad de microorganismos presentes

Tabla II. Distribución numérica y porcentual, por especies de microorganismos en aerosoles, en procedimientos clínicos con uso de pieza de mano de alta velocidad, en CODA, según el sitio de ubicación de placas prueba.

\begin{tabular}{lrrrrr}
\hline \multirow{2}{*}{ Especies } & \multicolumn{2}{c}{ Pechera } & \multicolumn{2}{c}{ Frente } & \multicolumn{1}{c}{ Total } \\
\hline Bacillus spp. & $\mathbf{n}$ & \multicolumn{1}{c}{$\%$} & \multicolumn{1}{c}{$\mathbf{n}$} & \multicolumn{1}{c}{$\%$} & \\
Bacilos Gram (+) & 241 & 25,58 & 28 & 2,97 & 26928,55 \\
Cocobacilos Gram (+) & 205 & 21,76 & 24 & 2,55 & 22924,31 \\
Corynebacterium spp. & 7 & 0,75 & 8 & 0,85 & 151,60 \\
Micrococcus spp. & 19 & 2,02 & 0 & 0,00 & 192,02 \\
Neisseria spp. & 115 & 12,21 & 26 & 2,76 & 14114,97 \\
Streptococos viridans & 4 & 0,42 & 0 & 0,00 & 40,42 \\
Stafilococos coagulasa (-) & 179 & 19,00 & 23 & 2,44 & 20221,44 \\
Streptococcus spp. & 33 & 3,50 & 19 & 2,01 & 525,52 \\
Total & 9 & 0,96 & 2 & 0,22 & 111,17 \\
& 812 & 86,20 & 130 & 13,80 & 942100 \\
\hline
\end{tabular}

Tabla III. Distribución numérica y porcentual, por especies de microorganismos en aerosoles, en procedimientos clínicos con uso de pieza de mano de alta velocidad, en CODA, según sexo.

\begin{tabular}{lrrrrr}
\hline \multirow{2}{*}{ Sexo } & \multicolumn{2}{c}{ Mujer } & \multicolumn{2}{c}{ Hombre } & \multirow{2}{*}{ Total } \\
\cline { 2 - 5 } Especies & \multicolumn{1}{c}{$\mathbf{n}$} & \multicolumn{1}{c}{$\%$} & \multicolumn{1}{c}{$\mathbf{n}$} & \multicolumn{1}{c}{$\%$} & \\
\hline Bacillus spp. & 217 & 23,04 & 52 & 5,51 & 26928,55 \\
Bacilos Gram + & 151 & 16,03 & 78 & 8,28 & 22924,31 \\
Cocobacilos Gram + & 5 & 0,53 & 10 & 1,07 & 151,60 \\
Corynebacterium spp. & 15 & 1,59 & 4 & 0,43 & 192,02 \\
Micrococcus spp. & 126 & 13,38 & 15 & 1,59 & 14114,97 \\
Neisseria spp. & 0 & 0 & 4 & 0,42 & 40,42 \\
Streptococos viridans & 134 & 14,22 & 68 & 7,22 & 20221,44 \\
Stafilococos coagulasa & 41 & 4,35 & 11 & 1,17 & 525,52 \\
Streptococcus spp. & 9 & 0,96 & 2 & 0,21 & 111,17 \\
Total & 698 & 74,10 & 244 & 25,90 & 942100 \\
\hline
\end{tabular}


en los aerosoles generados durante los procedimientos odontológicos en los que se usa turbina y constituyen un riesgo de infección para el paciente y el operador, lo que está en directa concordancia con estudios realizados por Shpuntoff \& Shpuntoff, Pankhurst, Prieto \& Maestre, Santiago et al. y Sanclement et al. entre otros.

En los análisis de control, no hubo crecimiento bacteriano procedente del medio ambiente, excepto en una de las ocho placas-control, donde se registró tres UFC de Micrococcus, bacteria que se encuentra en el suelo, agua y polvo del aire; la cual coloniza o puede ser parte de la flora normal de la piel y mucosas en los humanos, pero no se asocia con producción de infecciones lo que explicaría la presencia de este microorganismo, portado por el operador y/o paciente y transportado en el aire (Koneman), así como lo sitúa como un agente no potencialmente patológico al ser encontrado en el ambiente estudiado.

En las placas prueba, la mayoría de los microorganismos encontrados, son parte de la flora normal de la piel, como Streptococcus spp. viridans $(28,55 \%)$, Staphylococcus coagulasa negativa $(5,52 \%)$, Micrococcus $(14,97 \%)$. Un gran número de las especies de Streptococcus spp. $(1,17 \%)$ son patógenas, pero dependen del sitio de la infección. Se identificaron microorganismos de la orofaringe como Neisseria y Corynebacterium $(2,44 \%)$ y especies del aire, suelo, agua, como son Bacillus spp. $(28,55 \%)$, Bacilos Gram (+) (24.31\%) o Corynebacterium $(2,02 \%)$.

Lo relevante de estos resultados se traduce en el potencial patogénico de algunos de estos organismos ya que son capaces de producir infecciones en pacientes inmunodeprimidos, transformándose en patógenos oportunistas, tales como Corynebacterium, Bacillus, Staphylococcus coagulasa (-) y algunas especies de Streptococcus (Winn et al.).

Del mismo modo estos microorganismos pueden ser patógenos, dependiendo de la especie, por ejemplo: Bacillus anthracis, Corynebacterium diphtheriae, Neisseria meningitidis o gonorroheae. Los Staphylococcus coagulasa (-) son patógenos oportunistas, dependiendo de su especie, tales como Staphylococcus saprophyticus es patógeno en orina, causando infección urinaria. En esta investigación el análisis microbiológico sólo entregó descripción general de las especies encontradas, por lo que no se especificó de qué microorganismo específico se trata- ba (Dreyer \& Hauman). Por este motivo es difícil especificar si estábamos en presencia de microorganismos patógenos.

Desde este punto de vista, de los microorganismos identificados el Streptococcus seria el que causa mayor cantidad de infecciones en distintos lugares del cuerpo, por lo tanto, se prevee como el más patógeno, el resto de microorganismos serían comensales concordante con resultados obtenidos en experiencias similares, donde el promedio de UFC es comparable al encontrado en el estudio de Toroglu et al. (2001).

Desde el punto de vista de identificación de microorganismo según zona pechera de paciente o frente de operador, existe una diferencia marcada entre la cantidad de microorganismos encontrados en las ubicaciones descritas, siendo mayor la emisión de microorganismos hacia la pechera del paciente en concordancia con estudio de Pankhurst.

Existe una diferencia marcada entre la cantidad de microorganismos encontrados en los aerosoles recolectados desde la pechera del paciente y la frente del operador, siendo mayor la emisión de microorganismos hacia la pechera del paciente.

Al comprobar que los aerosoles constituyen una fuente importante de emisión de microorganismos, se hace imprescindible cumplir con todas las normas de bioseguridad que protegen tanto al operador como al paciente (Toroglu et al.; Harrel \& Molinari). Si bien es importante el uso de barreras para evitar el contacto directo con estos contaminantes, también es necesario higienizar cualquier superficie que haya estado en contacto con los aerosoles para evitar el contacto indirecto con los contaminantes, así como también la desinfección de los instrumentos dinámicos para evitar infecciones cruzadas.

Para eliminar el riesgo de infecciones cruzadas que representa la turbina de alta velocidad, el procedimiento de elección debe ser la esterilización por autoclave de esta, pero adecuándonos a la realidad del sistema docente asistencial recomendamos como medida mínima su desinfección externa con un germicida y hacerla funcionar con agua por 20 a 30 segundos antes de utilizarla, como indica los Centros para el Control y la Prevención de Enfermedades.

Se requieren más estudios acerca de este tema que involucren nuevas variables acerca del control de 
infecciones, la acción de distintos germicidas sobre la contaminación bacteriana de las superficies y del con- tenido patogénico de los aerosoles generados en odontología de manera más específica.

BUSTAMANTE, A. M. F.; HERRERA, M. J.; FERREIRA, A. R. \& RIQUELME, S. D. Analysis of Bacterial Contamination Produced by Aerosols in Dental Clinic Environments. Int. J. Odontostomat., 8(1):99-105, 2014.

ABSTRACT: In dental practice we are exposed to many microorganisms due to aerosol production. It is essential to be aware of the nature of these elements and their pathogenic potential. The purpose of this research is to determine the presence of bacterial contamination in aerosols produced during dental procedures that require the use of a high speed dental handpiece. These dental procedures were carried out by dentistry students at the dental clinic of the Universidad de La Frontera. In order to determine the presence of bacteria in aerosols, cultivation plates were used. A total of 40 samples were taken from 16 dentistry students while performing dental procedures that required the use of a high speed dental handpiece in conditions of complete isolation. Eight control plates and 32 test plates were used. Two selected areas were examined: operator's front and patient's chest protector, during 10 minutes. The samples were then taken to the laboratory and incubated in an oxygen atmosphere for 24 hours and subsequently in carbon dioxide for another 24 hours at a temperature of 37 degrees Celsius. Finally the samples were microscopically analyzed. Seven control plates showed no bacterial contaminants and one showed 3UFC of Micrococcus spp. The 32 test plates were found to be positive showing diverse bacterial growth (58.874 UFC average per subject). The highest percentage of microorganisms was constituted by Bacillus spp. (28.56 \%) and Bacillus Grampositivos (24.31\%), representing more than $50 \%$ of the total. The majority of the microorganisms found belong to the commensal family, although they can change into opportunistic pathogen microorganisms. Verifying that aerosols are an important source of emission of microorganisms, it is essential to comply with all biosecurity standards in order to protect the operator as well as the patient.

KEY WORDS: dental clinic environments, bacterial contamination, aerosols, turbine.

\section{REFERENCIAS BIBLIOGRÁFICAS}

Bentley, C. D.; Burkhart, N. W. \& Crawford, J. J. Evaluating spatter and aerosol contamination during dental procedures. J. Am. Dent. Assoc., 125(5):579-84, 1994.

Cascone, A. A.; Dolonguevich, E. R. \& Funes, S. A. Transmisión de la enfermedad periodontal en parejas estables a través del beso profundo. Rev. Fundac. Juan Jose Carraro, 7(16):13-8, 2002.

Checchi, L.; Matarasso, S.; Pirro, P. \& D'achille, C. Topographical analysis of the facial areas most susceptible to infection with transmissible diseases in dentists. Int. J. Periodontics Restorative Dent., 11(2):164-72, 1991.

de Beer, D.; Stoodley, P.; Roe, F. \& Lewandowski Z. Effects of biofilm structure on oxygen distribution and mass transport. Biotechnol. Bioeng., 43(11):1131-8, 1994.

de Lucas Tomas, M.; Velázquez Repeto, J. J. \& Iñiguez F. Microflora oral. Rev. Esp. Estomatol., 30(6):425-38, 1982.

Donlan, R. M. Biofilms: Microbial life on surfaces. Emerg. Infect. Dis., 8(9):881-90, 2002.

Dreyer, A. G. \& Hauman, C. H. Bacterial contamination of dental handpieces. SADJ, 56(11):510-2, 2001.

Grenier, D. Quantitative analysis of bacterial aerosols in two different dental clinic environments. Appl. Environ. Microbiol., 61(8):3165-8, 1995.

Hauman, C. H. Cross-infection risks associated with highspeed dental handpieces. J. Dent. Assoc. S. Afr., 48(7):389-91, 1993.

Harrel, S. K. \& Molinari, J. Aerosols and splatter in dentistry: a brief review of the literature and infection control implications. J. Am. Dent. Assoc., 135(4):429-37, 2004.

Loesche, W. J. Chemotherapy of dental plaque infections. Oral Sci. Rev., 9:65-107, 1976.

Molinares, J. A. Infection Control in a changing World. Operat. Infection Control, 1:1-8, 1993.

Pankhurst, C. L. Risk assessment of dental unit waterline contamination. Prim. Dent. Care, 10(1):5-10, 2003.

Prieto, J. \& Maestre, J. R. Tratamiento de las infecciones de etiología mixta. En: Bascones, A. \& Perea, E. J. (Eds). Infecciones Orofaciales. Madrid, Dentisnet, 2003. pp.245-56.

Sacchetti, R.; Baldissarri, A.; De Luca, G.; Lucca, P.; Stampi, S. \& Zanetti, F. Microbial contamination in dental unit waterlines: comparison between Er: YAG laser and turbine lines. Ann. Agric. Environ. Med., 13(2):275-9, 2006. 
BUSTAMANTE, A. M. F.; HERRERA, M. J.; FERREIRA, A. R. \& RIQUELME, S. D. Contaminación bacteriana generada por aerosoles en ambiente odontológico. Int. J. Odontostomat., 8(1):99-105, 2014.

Sanclement, J. A.; Webster, P. I.; Thomas, J. \& Ramadan, H. $\mathrm{H}$. Bacterial biofilms in surgical specimens of patients with chronic rhinosinusitis. Laryngoscope, 115(4):578-82, 2005.

Santiago, J. I.; Huntington, M. K.; Johnston, A. M. \& Williams, J. F. Microbial contamination of dental unit waterlines: short and long-term effects of flushing. Gen. Dent., 42(6):528-35, 1994

Shpuntoff, H. \& Shpuntoff, R. L. High-speed dental handpieces and spread of airborne infections. N. Y. State Dent. J., 59(1):21-3, 1993.

Toroglu, M. S.; Haytac, M. C. \& Köksal, F. Evaluation of Aerosol Contamination During Debonding Procedures. Angle Orthod., 71(4):299-306, 2001.

Williams, J. F.; Johnston, A. M.; Johnson, B.; Huntington, M. K. \& Mackenzie, C. D. Microbial contamination of dental unit waterlines: prevalence, intensity and microbiological characteristics. J. Am. Dent. Assoc., 124(10):59-65, 1993.

Winn, W.; Allen, S.; Janda, W.; Koneman, E.; Procop, G.; Schrenckenberger, P. \& Woods, G. Koneman. Diagnostico Microbiológico. $3^{\mathrm{a}}$ ed. Buenos Aires, Médica Panamericana, 1992.

Wood, P. R. Cross Infection Control in Dentistry. A Practical Illustrate Guide. St. Louis, Mosby Year Book, 1992. p.127.
Dirección para Correspondencia:

María Fabiola Bustamante Andrade

Departamento de Odontología Integral Adulto

Facultad de Odontología

Universidad de La Frontera

Casilla 54-D.

Montt $112,4^{\circ}$ piso

Temuco

CHILE

Fono: $56-045-2325775$

$56-045-2325766$

Email: fabiola.bustamante@ufrontera.cl

Recibido : 02-09-2013

Aceptado: 03-02-2014 